\title{
REVIEW
}

\section{Re-examining the efficacy of $\beta$-blockers for the treatment of hypertension: a meta-analysis}

\author{
Nadia Khan, Finlay A. McAlister
}

\section{ABSTRACT}

Background: In a recently published meta-analysis, investigators asserted that $\beta$-blockers should not be used to treat hypertension. Because the pathophysiology of hypertension differs in older and younger patients, we designed this metaanalysis to clarify the efficacy of $\beta$-blockers in different age groups. The primary outcome was a composite of stroke, myocardial infarction and death.

Methods: We identified randomized controlled trials that evaluated the efficacy of $\beta$-blockers as first-line therapy for hypertension in preventing major cardiovascular outcomes. Both authors independently evaluated the eligibility of all trials. Trials enrolling older (mean age at baseline $\geq$ 6 o years) patients were separated from those enrolling younger (mean age $<60$ years) patients. Data were pooled using a random effects model.

Results: Our analysis incorporated data from 145811 participants in 21 hypertension trials. In placebo-controlled trials, $\beta$-blockers reduced major cardiovascular outcomes in younger patients (risk ratio [RR] $0.86,95 \%$ confidence interval $[\mathrm{Cl}] 0.74-0.99$, based on 794 events in 19414 patients) but not in older patients (RR $0.89,95 \% \mathrm{Cl} 0.75^{-1.05}$, based on 1115 events in 8019 patients). In active comparator trials, $\beta$-blockers demonstrated similar efficacy to other antihypertensive agents in younger patients ( 1515 events in 30412 patients, RR $0.97,95 \% \mathrm{Cl} 0.88-1.07$ ) but not in older patients (7405 events in 79775 patients, RR 1.06, 95\% Cl 1.01-1.10), with the excess risk being particularly marked for strokes (RR 1.18, 95\% Cl 1.07-1.30).

Interpretation: $\beta$-blockers should not be considered firstline therapy for older hypertensive patients without another indication for these agents; however, in younger patients $\beta$ blockers are associated with a significant reduction in cardiovascular morbidity and mortality.

CMAJ 2006;174(12):1737-42

영 $\mathrm{n}$ a recent and widely publicized meta-analysis by Lindholm and colleagues, ${ }^{1}$ data were pooled from 18 randomized trials in which $\beta$-blockers were used as firstline treatment for hypertension. The authors found an excess risk of stroke associated with $\beta$-blockers compared with other classes of antihypertensive drugs, and they concluded that $\beta$-blockers should not remain one of the first- choice options for the treatment of hypertension. ${ }^{1}$ The accompanying editorial stated that "the era of $\beta$-blockers for hypertension is over." ${ }^{2}$ However, we believe this conclusion is flawed for several reasons. ${ }^{3}$

First, not all randomized hypertension trials relevant to this question were included in the meta-analysis - in particular, several large trials that have been included in other hypertension meta-analyses were excluded. ${ }^{4-6}$ Second, the investigators focused on an individual end point (stroke), rather than the composite outcome, usually reported in hypertension clinical trials, of "major cardiovascular outcomes" (stroke, myocardial infarction [MI] or death). When an individual end point, such as stroke, is used, an agent with a beneficial impact on some end points may thus appear to have a detrimental effect on another individual end point because of survivor bias (e.g., if an agent prevented coronary deaths, then more patients given that agent would be alive and at risk of stroke than patients given another agent that did not reduce coronary deaths). It is important to note that, contrary to the perception fostered by the meta-analysis, the most common events in the hypertension trials analyzed were death $(n=8909)$ and MI $(n=4701)$, not strokes $(n=4337)$ or heart failure $(n=\mathrm{I} 93 \mathrm{I})$.

Third, the data used was both statistically $(p=0.02)$ and clinically heterogeneous. In such a situation, pooled estimates can be misleading, and analyses within homogeneous patient subgroups may be more appropriate than simply pooling all of the trial data together. Since hypertension in elderly patients is characterized by low arterial compliance and increased vascular resistance, and hypertension in younger patients is characterized by a high cardiac output in the face of normal or reduced peripheral vascular resistance, ${ }^{7,8}$ it is not unreasonable to speculate that drugs that reduce cardiac output without affecting vessel wall compliance (such as $\beta$-blockers) ${ }^{9}$ will demonstrate different effects in older hypertensive patients. As such, we felt it inappropriate to pool data from trials involving people in their 70 s and 80 s with those from trials involving people in their 405 and $50 s$.

Thus, in the present meta-analysis, we sought to clarify the efficacy of $\beta$-blockers for primary hypertension with analyses that incorporate the data from all relevant $\beta$-blocker trials, using the composite outcome of major cardiovascular events (stroke, MI or death) relevant to hypertension patients and their physicians. Most importantly, we sought to explore and explain the heterogeneity in the $\beta$-blocker trial results by comparing outcomes in younger and older patients. 


\section{Methods}

We conducted a search on PubMed (I950 to Jan. I8, 2006) using the search terms " $\beta$ blockers or adrenergic $\beta$ antagonists" and "hypertension" and "stroke or death or myocardial infarction," restricted to humans and clinical trials. We also reviewed reference lists from previous hypertension meta-analyses identified by searches of MEDLINE and the Cochrane Library, and contacted Canadian hypertension experts. We included only randomized controlled trials that evaluated the efficacy of $\beta$-blockers as first-line therapy for hypertension in preventing major cardiovascular events (stroke, MI or death). We included open randomized trials (as long as there was blinded ascertainment of the outcomes in the trial) and randomized trials that assigned patients to "mixed older agents" as one of the treatment arms as long as at least $50 \%$ of the patients receiving the "mixed older agents" received a $\beta$-blocker (in sensitivity analyses we explored results with and without inclusion of these mixed trials).

Both authors extracted outcome data from each trial independently; outcomes were assigned according to the intention-to-treat principle and using the outcome definitions employed in each study (the end point definitions and methods of classification were identical across treatment groups within each trial). Our primary outcome was the composite cardiovascular outcome of death, nonfatal MI or nonfatal stroke, a clinically relevant end point that was elected after discussion by 44 national hypertension experts at the Canadian Hypertension Education Program consensus conference on Oct. 2I, 2005. We also explored outcomes for heart failure as well as for each component of the composite outcome separately. Because we did not have access to individual patient data, we used trial inclusion criteria or the mean age of trial participants or both to distinguish between trials enrolling "younger" $(<60$ years of age) patients and those enrolling "older" ( $\geq 60$ years of age) patients. Trials were dichotomized using a patient age of 60 years to be consistent with prior reports in this field. ${ }^{10}$

Meta-analyses for all outcomes were performed using random-effects models, and $\chi^{2}$ tests for heterogeneity were used to assess between-study heterogeneity for each outcome analysis. Pooled risk ratios (RR) were expressed with $95 \%$ confidence intervals (CIs). Sensitivity analyses were performed to explore the outcomes after excluding those trials with "mixed older agents" as one treatment arm (in which $\beta$ blockers were only one of the first-line options in that arm of the study) as well as the outcomes in those trials included in the meta-analysis by Lindholm and colleagues.

\section{Results}

Our search results are outlined in online Appendix I, available at www.cmaj.ca/cgi/content/full/174/12/1737/DCr. The interobserver kappa for trial inclusion was 0.94. In contrast to the meta-analysis by Lindholm and associates, we included the data from the Captopril Prevention Project (CAPPP) ${ }^{11}$ the Veterans Administration Cooperation Study Group Trial, ${ }^{12}$ and the African American Study of Kidney Disease and Hyperten- sion (AASK) $)^{13}$ in our analysis since patients in all 3 trials were randomly assigned to $\beta$-blockers as initial therapy in at least one of the treatment arms, and all 3 trials have been included in previous hypertension systematic reviews. ${ }^{4-6}$ As with to the meta-analysis by Lindholm and associates and other hypertension meta-analyses, ${ }^{1,4}$ we excluded the results from the Metoprolol Atherosclerosis in Hypertension (MAPHY) trial ${ }^{14}$ because this study was a follow-up extension of a subgroup from the Heart Attack Primary Prevention in Hypertension (HAPPHY) trial. ${ }^{15}$

We collected outcome data on I45 8II participants in 2I hypertension $\beta$-blocker trials ${ }^{11-13,15-32}$ published between 1982 and 2005. Of these, 6 trials employed placebo control subjects in at least one arm of the study (online Appendix 2, www.cmaj .ca/cgi/content/full/174/12/1737/DC2). Although most of these trials included $\beta$-blocker monotherapy as one of the randomization arms, in 5 trials the assignment was to "mixed older agents" versus other antihypertensive drug classes. ${ }^{11,16-19}$ The actual proportion of patients randomly assigned to "mixed older agents" who received $\beta$-blocker monotherapy at randomization in these 5 trials ranged from $54 \%$ to $75 \%$. The mean age ranged from 45.5 to 56.2 years in the trials enrolling younger patients ( $n=10$, with 50612 patients) and from 60.4 to 76 years in the trials enrolling older patients $(n=\mathrm{II}$, with 95 I99 patients).

\section{Placebo-controlled trials}

With regard to the composite outcome (death, stroke or MI), $\beta$-blockers reduced event rates compared with placebo ( $R R$ 0.86 , 95\% CI 0.74-0.99, based on 794 events in I9 $4 \mathrm{I} 4 \mathrm{pa}-$ tients) in trials enrolling younger patients (Fig. IA), ${ }^{21,28}$ but benefits were not found in trials enrolling older patients $^{19,24,29-31}$ (RR 0.89, 95\% CI 0.75-I.05, based on III5 events in 8org patients) (Fig. IB). There was no evidence of heterogeneity in these age-specific pooled analyses.

$\beta$-blockers were associated with trends toward reduced rates of MI (RR 0.85, 95\% CI 0.7I-I.03), stroke (RR 0.84, 95\% CI 0.65-I.Io), and death (RR 0.94, 95\% CI 0.79-I.IO), but not heart failure (RR I.05, 95\% CI 0.72-I.54) in placebocontrolled trials enrolling younger patients. None of these individual end points occurred frequently enough to permit definitive conclusions to be drawn, given insufficient power in these analyses. In trials involving older patients, $\beta$-blockers were associated with statistically significant reductions in stroke (RR $0.78,95 \% \mathrm{CI} 0.63-0.98$ ) and heart failure (RR $0.54,95 \%$ CI o.37-0.8I) compared with placebo, but had no appreciable impact on rates of MI (RR 0.98 , 95\% CI o.83I.I6) or death (RR 0.9I, 95\% CI 0.74-I.I2).

\section{Active comparator trials}

With regard to the composite outcome (death, stroke or MI) among $304 \mathrm{I} 2$ patients younger than 60 years of age,,$^{11,15,21-23}$ there was no difference in event rates between those randomly assigned to $\beta$-blocker therapy compared with those receiving other antihypertensive agents (1515 events, RR 0.97, 95\% CI o.88-I.07) (Fig. 2A). However, in the 79775 patients 
60 years of age or older, ${ }^{16-18,24-27} \beta$-blockers were associated with a higher risk of events than were other antihypertensive agents (7405 events, RR I.06, 95\% CI I.OI-I.Io) (Fig. 2B). Importantly, there was no evidence for heterogeneity in these pooled analyses.

In randomized comparisons with other antihypertensive agents in younger patients, $\beta$-blockers exhibited similar efficicacy for the individual end points of MI (RR 0.97, 95\% CI 0.86-I.Io), death (RR $0.97,95 \%$ CI 0.83 -I.I4), heart failure (RR $0.93,95 \% \mathrm{CI}$ $0.64-\mathrm{I} .34$ ) and stroke (RR 0.99 , $95 \%$ CI o.67-I.44). However, in trials involving older patients, $\beta$ blockers were associated with significantly higher rates of stroke (2935 strokes in 87 I80 patients, RR I.I8, 95\% CI I.07-I.30, $p$ for heterogeneity $=0 . \mathrm{II})$, but not MI (RR I.06, 95\% CI 0.94-I.20), heart failure (RR 0.98, 95\% CI o.87-I.II), or death (RR I.05, 95\% CI 0.99-I.II).

\section{Sensitivity analyses}

In an analysis excluding the 5 trials with "mixed older agents" as one of the randomization arms (in which outcome data for patients given $\beta$ blockers were reported in combination with the outcome data for patients given thiazides), our results were unchanged: in the trials involving younger patients, there was no difference in composite event rates between those randomly assigned to $\beta$-blockers and those assigned to other antihypertensive agents (RR I.oI, 95\% CI 0.88-I.I5), but in older patients $\beta$-blockers were associated with a higher risk of the composite outcome than were other antihypertensive agents (RR I.I2, 95\% CI I.02-I.24). When we excluded the 3 studies excluded from the meta-analysis by Lindholm and associates, the results were consistent with those found in our main analysis described above: no difference in composite event rates in trials of active comparators involving younger patients (RR I.OI, 95\% CI o.88-I.I5), but excess risk in active comparator trials involving older patients (RR I.07, 95\% CI I.00-I.I4) that was largely driven by excess risk of stroke (RR I.I8, 95\% CI I.07-I.30).

\section{Interpretation}

Our results confirm the finding in the meta-analysis by Lindholm and colleagues that $\beta$-blockers are associated with an increased risk of stroke compared with other antihypertensive agents, but the results also show that this excess risk is largely driven by data from trials enrolling older patients. Im-

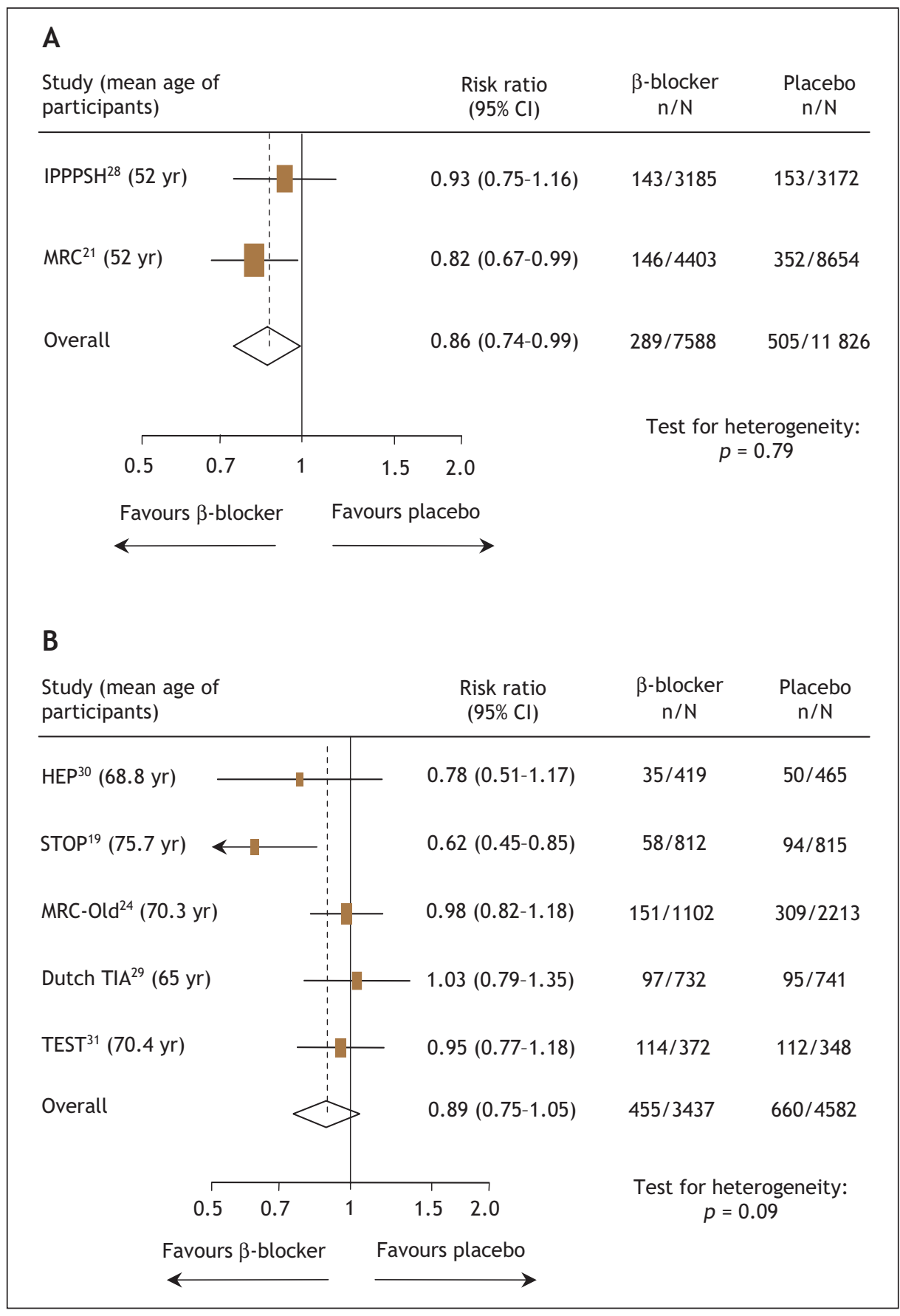

Fig. 1: Risk ratios for the composite outcome (death, stroke or myocardial infarction) for (A) patients less than 60 years of age and (B) patients 60 years of age and older receiving $\beta$ blockers or placebo. The size of the boxes represents the number of participants who experienced a cardiovascular event. The mean age of trial participants is given in parentheses after each trial acronym. Trials are listed in order of publication. $\mathrm{Cl}=$ confidence interval. 
portantly, younger patients randomly assigned to $\beta$-blockers exhibit similar rates of cardiovascular death, MI or stroke to those assigned other antihypertensive agents, and $\beta$-blockers were more efficacious than placebo in these patients.
Our analysis supports the cautions raised almost a decade ago by Messerli and colleagues that $\beta$-blockers are a poor initial choice for first-line therapy for uncomplicated hypertension in elderly patients. ${ }^{10}$ However, we have expanded upon

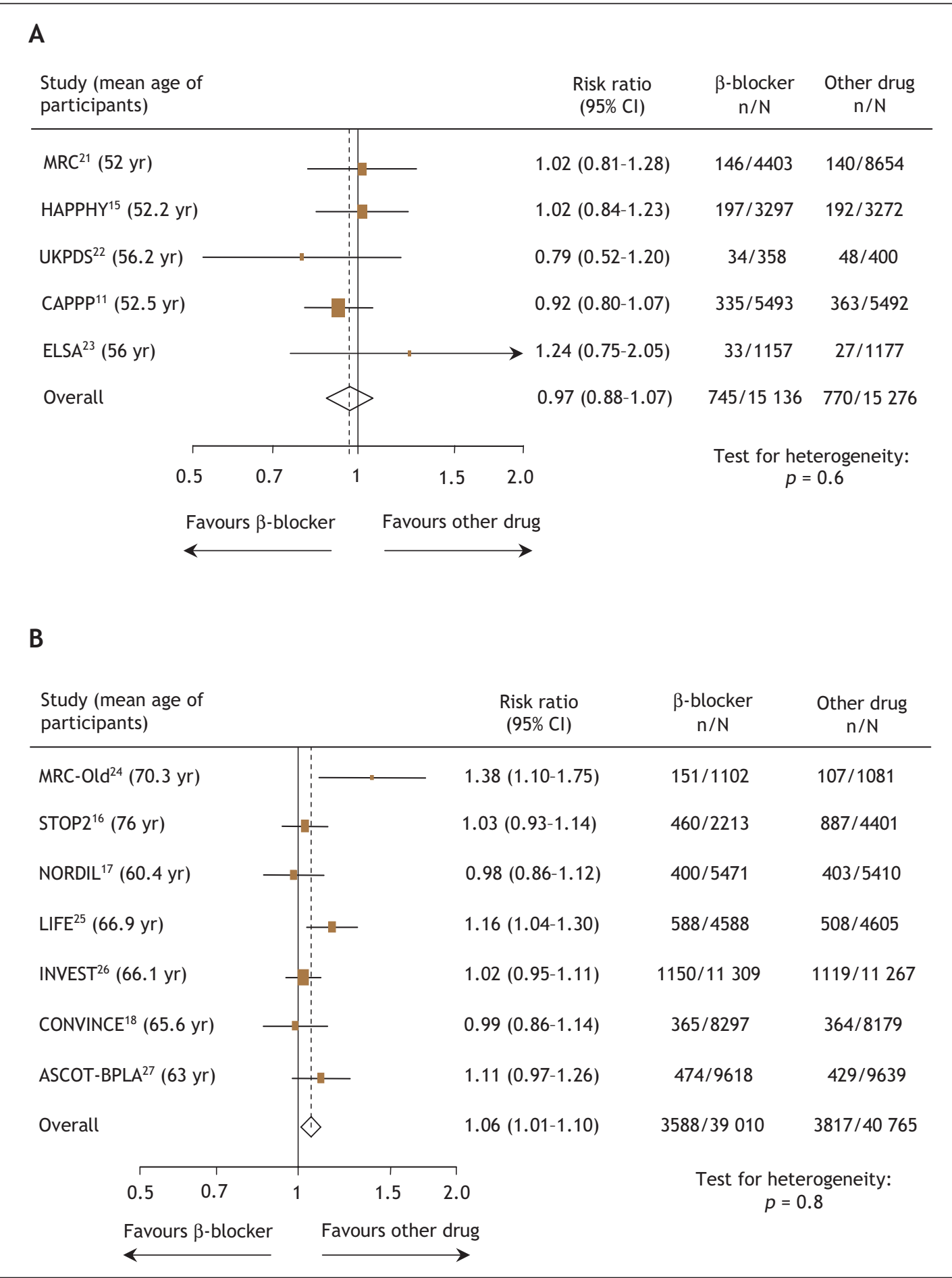

Fig. 2: Risk ratios for the composite outcome (death, stroke or myocardial infarction) for (A) patients less than 60 years of age and $(B)$ patients 60 years of age and older receiving $\beta$-blockers or other antihypertensive drugs. The size of the boxes represents the number of participants who experienced a cardiovascular event. The mean age of trial participants is given in parentheses after each trial acronym. Trials are listed in order of publication. $\mathrm{Cl}=$ confidence interval. 
their analysis by including more recently published data from an additional I4I II7 patients in I8 trials. Although our analysis demonstrates that $\beta$-blockers are beneficial in younger hypertensive patients, it should be acknowledged that the observed benefits in the placebo-controlled trials are less than might be expected given the results of epidemiologic studies. ${ }^{33}$ Although it remains unclear whether certain classes of antihypertensive drugs have greater or lesser impact on causespecific cardiovascular outcomes or in different patient subgroups (e.g., black patients), the results of a network metaanalysis of 42 trials (incorporating trials comparing agents from all drug classes) suggested that low-dose thiazide diuretics were the most effective first-line therapy for hypertension. ${ }^{4}$ However, in those younger patients with contraindications or prior intolerance to thiazide diuretics, our analysis supports the use of $\beta$-blockers as a first-line agent for lowering blood pressure.

Like all meta-analyses, our analysis does have some limitations. Given the paucity of data in the published reports for each trial, we cannot adjust our analyses for degree of blood pressure control, dose of medications nor compliance with assigned therapy. Similarily, since virtually none of the trials reported data in age-specific subgroups, we had to extrapolate from the trial eligibility criteria and the mean age of study participants to divide the trials into those enrolling younger patients and those enrolling older patients. However, although the ongoing Blood Pressure Lowering Treatment Trialists Collaboration ${ }^{5}$ is prospectively collecting the individual patient data necessary to fully explore outcomes in age-specific subgroups, their ability to explore the efficacy of $\beta$-blockers in different age groups is limited by the fact that the Collaboration is only collecting data on trials started after 1995 . This thereby excludes much of the data used in our analysis, and, given the decreasing enthusiasm for $\beta$-blocker monotherapy as the active comparator in future trials, it is questionable whether further data will be available in the future.

Thus, although $\beta$-blockers should clearly not be considered first-line therapy for older hypertensive patients without another indication (such as heart failure, ${ }^{34}$ postmyocardial infarction ${ }^{35}$ or symptomatic coronary disease ${ }^{36}$ ), in younger patients $\beta$-blockers are more efficacious than placebo, and there is robust evidence from trials enrolling over 30 ooo hypertensive patients to refute the claim that $\beta$-blockers are less beneficial than other antihypertensive agents. Our analysis supports the stance espoused in the 2006 Canadian Hypertension Education Program Recommendations ${ }^{37}$ that $\beta$-blockers should remain one of the recommended drug classes in the therapeutic armamentarium for younger hypertensive patients. In the editorial accompanying the meta-analysis by Lindholm and associates, Beevers cautioned that there was a danger of "throwing out the baby with the bath water" in recommending against the use of $\beta$-blockers for the treatment of hypertension. ${ }^{2}$ We agree.

\section{This article has been peer reviewed.}

From the Division of General Internal Medicine, University of British Columbia (Khan),Vancouver, BC, and the Division of General Internal Medicine, University of Alberta (McAlister), Edmonton, Alta.
Competing interests: None declared.

Contributors: Both authors contributed equally to the conception, design, conduct, analysis and writing of this article, and both have approved the final version for publication.

Acknowledgements: We would like to thank Dr. Richard Z. Lewanczuk for his thoughtful insights on the pathophysiology of hypertension according to age.

Nadia Khan is a GENESIS (Canadian Institutes of Health Research [CIHR] and Heart and Stroke Foundation of Canada) Young Investigator and a St. Paul's Hospital Foundation Research New Investigator. Finlay McAlister is supported by the Alberta Heritage Foundation for Medical Research, CIHR and the University of Alberta/Merck Frosst/Aventis Chair in Patient Health Management.

\section{REFERENCES}

I. Lindholm LH, Carlberg B, Samuelsson O. Should $\beta$-blockers remain first choice in the treatment of primary hypertension? A meta-analysis. Lancet 2005;366:1545-53.

2. Beevers DG. The end of beta blockers for uncomplicated hypertension? Lancet 2005;366:1510-2.

3. Khan N, McAlister FA. $\beta$ Blockers for the treatment of primary hypertension [letter]. Lancet 2006;367:208.

4. Psaty BM, Lumley T, Furberg CD, et al. Health outcomes associated with various antihypertensive therapies used as first-line agents a network meta-analysis. JAMA 2003;289:2534-44.

5. Blood Pressure Lowering Trialists' Collaboration. Effects of different blood-pressure-lowering regimens on major cardiovascular events: results of prospectively designed overviews of randomised trials. Lancet 2003;362:1527-35.

6. Staessen JA, Wang JG, Thijs L. Cardiovascular prevention and blood pressure reduction: a quantitative overview updated until I March 2003. J Hypertens 2003;2I: 1055-76.

7. Benetos A, Waeber B, Izzo J, et al. Influence of age, risk factors, and cardiovascular and renal disease on arterial stiffness: Clinical applications. Am J Hypertens 2002; I5:IIOI-8

8. Franklin SS, Gustin W, Wong ND, et al. Hemodynamic patterns of age-related changes in blood pressure. The Framingham Heart Study. Circulation 1997;96: 308-I5.

9. Resnick LM, Lester MH. Differential effects of antihypertensive drug therapy on arterial compliance. Am J Hypertens 2002;15:I096-Ioo.

Io. Messerli FH, Grossman E, Goldbourt U. Are $\beta$-blockers efficacious as first-line therapy for hypertension in the elderly? JAMA 1998;279:1903-7.

II. Hansson L, Lindholm LH, Niskanen L, et al. Effect of angiotensin-converting-enzyme inhibition compared with conventional therapy on cardiovascular morbidity and mortality in hypertension: the Captopril Prevention Project (CAPPP) randomised trial. Lancet I999;353:611-6.

I2. Veterans Administration Cooperative Study Group on Antihypertensive Agents. Comparison of propranolol and hydrochlorothiazide for the initial treatment of hypertension. II. Results of long-term therapy. JAMA I982;248:2004-II.

I3. Wright JT, Bakris G, Greene T, et al. Effect of blood pressure lowering and antihypertensive drug class on progression of hypertensive kidney disease. Results from the AASK Trial. JAMA 2002;288:242I-3I.

I4. Wikstrand J, Warnold I, Olsson G, et al. Primary prevention with metoprolol in patients with hypertension. JAMA I988;259:1976-82.

I5. Wilhelmsen L, Berglund G, Elmfeldt D, et al. Beta-blockers versus diuretics in hypertensive men: main results from the HAPPHY trial. J Hypertens I987;5:56I-72.

I6. Hansson L, Lindholm LH, Ekbom T, et al. Randomised trial of old and new antihypertensive drugs in elderly patients: cardiovascular mortality and morbidity in the Swedish Trial in Old Patients with Hypertension-2 study. Lancet 1999;354:175I-6.

I7. Hansson L, Hedner T, Lund-Johansen P, et al. Randomised trial of effects of calcium antagonists compared with diuretics and $\beta$ blockers on cardiovascular morbidity and mortality in hypertension: the Nordic Diltiazem (NORDIL) study. Lancet 2000;356:359-65.

I8. Black HR, Elliott WJ, Grandits G, et al. Principal results of the Controlled Onset Verapamil Investigation of Cardiovascular End Points (CONVINCE) Trial. JAMA 2003;289:2073-82.

I9. Dahlöf B, Lindholm LH, Hansson L, et al. Morbidity and mortality in the Swedish Trial in Old Patients with hypertension (STOP-Hypertension). Lancet I991;338: I28I-5.

20. Berglund G, Andersin O, Widgren B. Low-dose antihypertensive treatment with a thiazide diuretic is not diabetogenic. Acta Med Scand I986;220:419-24.

2I. MRC trial of treatment of mild hypertension: principal results. Medical Research Council Working Party. BMJ I985;291:97-104

22. UK Prospective Diabetes Study Group. Efficacy of atenolol and captopril in reducing risk of macrovascular and microvascular complications in type 2 diabetes: UKPDS 39. BMJ1998;317:713-20.

23. Zanchetti A, Bond MG, Henning $M$, et al. Calcium antagonist lacidipine slows down progression of asymptomatic carotid atherosclerosis. Principal results of the European lacidipine study on atherosclerosis (ELSA), a randomized, double-blind, long-term trial. Circulation 2002;106:2422-7.

24. MRC Working Party. Medical Research Council trial of treatment of hypertension 
in older adults: principal results. BMJ 1992;304:405-12.

25. Dahlöf B, Devereux RB, Kjeldsen SE, et al. Cardiovascular morbidity and mortality in the losartan intervention for endpoint reduction in hypertension study (LIFE): a randomised trial against atenolol. Lancet 2002;359:995-1003.

26. Pepine CJ, Handberg EM, Cooper-DeHoff RM, et al. A calcium antagonist vs a non-calcium antagonist hypertension treatment strategy for patients with coronary artery disease. The International Verapamil-Trandolapril Study (INVEST): a randomized controlled trial. JAMA 2003;290:2805-16.

27. Dahlöf B, Sever PS, Poulter NR, et al. Prevention of cardiovascular events with an antihypertensive regimen of amlodipine adding perindopril as required versus atenolol adding bendroflumethiazide as required, in the Anglo-Scandinavian Cardiac Outcomes Trial-Blood Pressure Lowering Arm (ASCOT-BPLA): a multicentre randomised controlled trial. Lancet 2005;366:895-906.

28. The IPPPSH Collaborative Group. Cardiovascular risk and risk factors in a randomised trial of treatment based on the beta-blocker oxprenolol: The international prospective primary prevention study in hypertension (IPPPSH). J Hypertens 1985 3:379-92.

29. Trial of secondary prevention with atenolol after transient ischemic attack or nondisabling ischemic stroke. The Dutch TIA Trial Study Group. Stroke I993;24: $543-8$.

30. Coope J, Warrender TS. Randomised trial of treatment of hypertension in elderly patients in primary care. $B M J$ I986;293:1145-5I.

31. Eriksson S, Olofsson B-O, Wester P-O, for the TEST Study Group. Atenolol in secondary prevention after stroke. Cerebrovasc Dis 1995;5:2I-5.

32. Yurenev AP, Dyakonova HG, Novikov ID, et al. Management of essential hypertension in patients with different degrees of left ventricular hypertrophy. Multicenter trial Am J Hypertens 1992;6:182S-9S.

33. Collins R, Peto R, MacMahon S, et al. Blood pressure, stroke, and coronary heart disease. Part 2, Short-term reductions in blood pressure: overview of randomised drug trials in their epidemiological context. Lancet 1990;335:827-38.

34. Brophy JM, Joseph L, Rouleau JL. Beta-blockers in congestive heart failure. A Bayesian meta-analysis. Ann Intern Med 2001;134:550-60.

35. The Beta-Blocker Pooling Project (BBPP): subgroup findings from randomized trials in post-infarction patients. The Beta-Blocker Pooling Project Research Group Eur Heart J I988;9:8-I6.

36. Heidenreich PA, McDonald KM, Hastie T, et al. Meta-analysis of trials comparing beta-blockers, calcium antagonists, and nitrates for stable angina. JAMA I999;28I 1927-36.

37. Khan NA, McAlister FM, Rabkin SW, et al.; Canadian Hypertension Education Program. The 2006 Canadian Hypertension Education Program (CHEP) recommendations for the management of hypertension: Part 2 - Therapy. Can J Cardiol. In press.

Correspondence to: Dr. Finlay McAlister, $2 E_{3} .24$ Walter Mackenzie Health Sciences Centre, University of Alberta Hospital, 8440 II2 St., Edmonton AB T6G 2R7

\section{ACCESS}

$C M A J$ is the only leading general medical journal that is free online. CMAJ.ca receives over 2 million hits per month of which two-thirds are from international readers. 